\title{
Chaos control of the modified Chua's circuit system
}

\author{
Tzuyin $\mathrm{Wu}^{*}$, Min-Shin Chen \\ Department of Mechanical Engineering, National Taiwan University, Taipei 106, Taiwan \\ Received 22 December 2000; accepted 12 January 2002 \\ Communicated by M. Sano
}

\begin{abstract}
In this paper, a nonlinear controller called the backstepping controller is applied to suppress the chaotic motion of a modified Chua's circuit system. The new controller can drive the system to the exact reference state at any prescribed speed. Most importantly, the controller achieves global exponential stability in the sense that the attraction basin for the reference state is the entire state space. Previous controllers for the Chua's circuit can achieve only local stability in the sense that the attraction basin is a subset of the state space. (C) 2002 Elsevier Science B.V. All rights reserved.
\end{abstract}

PACS: $05.45 .+\mathrm{b}$

Keywords: Chaos control; Chua's circuit; Nonlinear control; Backstepping control

\section{Introduction}

In the last decade, much effort has been devoted to the study of nonlinear chaotic systems. As more and more knowledge is gained about the nature of chaos, recent interests are now directed to controlling a chaotic system; that is, bringing the chaotic state to a fixed point or a limit cycle. In the literature, there are two basic approaches to the control of a chaotic system: non-feedback control, and feedback control. For the non-feedback control, it is demonstrated that a small periodic parametric perturbation can tame the chaos [1,2]. For the feedback control, there are two different methods: one is the data-based control, and the other model-based control. For the data-based feedback control, knowledge of the system model is not required. A time-varying small parametric perturbation is created based on a discrete feedback of the delay-coordinate vector of a state variable [3,4]. Such a control can bring the chaotic system state to an originally unstable periodic orbit which is embedded in the chaotic attractor. For the model-based feedback control, a linear or nonlinear feedback controller is designed based on the full knowledge of the system model. This kind of control has the capability to reshape the whole state space trajectory, and to create new stable fixed points or limit cycles $[5,6]$.

In this paper, we consider the feedback control of a modified Chua's circuit. The Chua's circuit is an extensively studied and well-understood chaotic system [7]. The original Chua's circuit contains a piecewise linear resistor. Hartley [8] suggested that the piecewise linear function can be replaced by a cubic polynomial with no major

* Corresponding author. Tel.: +886-2-236-30231x2413; fax: +886-2-236-31755.

E-mail address: tywu@ @cms.ntu.edu.tw (T. Wu). 
change in the system's dynamic characteristics. For the (modified) Chua's circuit, there have been several controllers proposed to suppress the chaos. Hartley and Mossayebi [9] used the $L Q$ optimal state feedback control and a classical lead compensator to drive the state to a desired fixed point on the equilibrium manifold. Hwang et al. [10] proposed a different linear state feedback control to regulate the system state, claiming faster settling time. Chen and Dong [11] used proportional control to drag the system state out of the strange attractor, and into an unstable limit cycle of the Chua's circuit.

However, since the chaotic system is nonlinear, the above "linear" controls [3,4,9-11] do not guarantee stability or performance in the global sense. If the initial state of the system lies outside the attraction basin, the state trajectory will not converge to the target. Therefore, one has to resort to nonlinear control in order to obtain global results. In this paper, a nonlinear backstepping controller [12] is adopted to control the modified Chua's circuit. Even though this new controller has a more complicated structure than previous ones, it has the advantage that the attraction basin of the desired fixed point is the whole state space. In other words, the controlled system is globally stable. Furthermore, it is always possible to tune the parameters in the backstepping controller such that the convergence to the target is as fast as desired.

\section{Backstepping control}

Consider the modified Chua's circuit system proposed in [8]:

$$
\begin{aligned}
& \dot{x}=p(y-f(x)), \quad f(x)=\frac{2 x^{3}-x}{7}, \\
& \dot{y}=x-y+z, \\
& \dot{z}=-q y+u,
\end{aligned}
$$

where $p=10, q=100 / 7$ are positive system parameters, the control input $u$ represents a voltage source in series with the inductor, $x$ and $y$ are voltages across two capacitors, and $z$ is the current through the inductor.

Without the control $u$, the modified Chua's circuit is known to generate a chaotic phenomenon when the system parameters are set at values specified above. In this section, a backstepping control will be introduced to suppress this chaotic motion. Furthermore, the proposed control will bring the system state, say $x$, to any reference state $x_{\mathrm{r}}$ at any prescribed speed. The proposed control is as follows:

$$
\begin{aligned}
u= & a(x) x+b(x) y+c(x) z+d(x) y^{2}+e f(x)+g f(x) f^{\prime}(x)+p^{2} f(x) f^{\prime}(x)^{2} \\
& +p^{2} f^{2}(x) f^{\prime \prime}(x)+\frac{1}{8 p} k_{x} k_{y} k_{z} x_{\mathrm{r}},
\end{aligned}
$$

where

$$
\begin{aligned}
& a(x)=1-\frac{1}{2}\left(k_{x}+k_{y}+k_{z}\right)-\frac{1}{8 p} k_{x} k_{y} k_{z}+p f^{\prime}(x), \\
& b(x)=q-p-1+\frac{1}{2}\left(k_{x}+k_{y}+k_{z}\right)-\frac{1}{4}\left(k_{x} k_{y}+k_{y} k_{z}+k_{z} k_{x}\right) \\
& -\left(1-\frac{1}{2}\left(k_{x}+k_{y}+k_{z}\right)\right) p f^{\prime}(x)-p^{2} f^{\prime}(x)^{2}-2 p^{2} f(x) f^{\prime \prime}(x), \\
& c(x)=1-\frac{1}{2}\left(k_{x}+k_{y}+k_{z}\right)+p f^{\prime}(x), \quad d(x)=p^{2} f^{\prime \prime}(x), \\
& e=p+\frac{1}{4}\left(k_{x} k_{y}+k_{y} k_{z}+k_{z} k_{x}\right), \quad g=-\frac{p}{2}\left(k_{x}+k_{y}+k_{z}\right),
\end{aligned}
$$


and $k_{x}, k_{y}$ and $k_{z}$ are three positive control gains, which determine the convergence rate of the controlled system. The backstepping controller is somewhat more complicated than previous controllers; however, the added complexity allows one to claim global stability of the controlled system, and to have exact control over how fast the system state converges.

\section{Stability analysis}

The design philosophy of the proposed controller (4) is based on the backstepping principle [12]. The backstepping controller design, in our case, consists of three steps:

Step I: In the $x$-state Eq. (1), one treats the second state variable $y$ as a control input, and finds out the desired $y$, say $y=y_{\mathrm{d}}\left(x, x_{\mathrm{r}}\right)$ that will force $x$ to converge to the reference state $x_{\mathrm{r}}$ at a prescribed speed.

Step II: In the $y$-state Eq. (2), one treats the third state variable $z$ as a control input, and finds out the desired $z$, say $z=z_{\mathrm{d}}\left(x, y, x_{\mathrm{r}}\right)$ that will force $y$ to approach the previously designed function $y_{\mathrm{d}}$ at a prescribed speed.

Step III: Finally, in the $z$-state Eq. (3), one chooses the true control input $u$ to drive $z$ to approach the previously designed function $z_{\mathrm{d}}$ at a prescribed speed.

The detailed design procedures and stability analysis are presented below. Firstly, in the design procedure Step I, the desired $y_{\mathrm{d}}$ function is chosen as

$$
y_{\mathrm{d}}\left(x, x_{\mathrm{r}}\right)=f(x)-\frac{k_{x}}{2 p}\left(x-x_{\mathrm{r}}\right) .
$$

Lemma 1. If the second state variable $y$ in Eq. (1) is equal to $y_{\mathrm{d}}$, the first state variable $x$ will approach $x_{\mathrm{r}}$ exponentially fast.

Proof. Define a Lyapunov function candidate $V_{x}=\frac{1}{2}\left(x-x_{\mathrm{r}}\right)^{2} \geq 0$. From Eqs. (1) and (5), the time derivative of $V_{x}$ is found to be $\dot{V}_{x}=-k_{x} V_{x}$. Consequently, $x(t)$ approaches $x_{\mathrm{r}}$ exponentially fast in the following way:

$$
\left|x(t)-x_{\mathrm{r}}\right|=\left|x(0)-x_{\mathrm{r}}\right| \mathrm{e}^{-\left(k_{x} / 2\right) t}
$$

Secondly, in the design procedure Step II, the desired $z_{\mathrm{d}}$ function is chosen as

$$
z_{\mathrm{d}}\left(x, y, x_{\mathrm{r}}\right)=-\left(1+\frac{k_{x} k_{y}}{4 p}\right) x+\left(1-\frac{k_{y}+k_{x}}{2}\right) y+\frac{k_{y}+k_{x}}{2} f(x)+\frac{k_{x} k_{y}}{4 p} x_{\mathrm{r}}+p f^{\prime}(x)(y-f(x)) .
$$

Lemma 2. If the third state variable $z$ in Eq. (2) is equal to $z_{\mathrm{d}}$, the second state variable $y$ will approach $y_{\mathrm{d}}$ exponentially fast.

Proof. Define a second Lyapunov function candidate $V_{y}=\frac{1}{2}\left(y-y_{\mathrm{d}}\right)^{2} \geq 0$. From Eqs. (2) and (6), one can verify that the time derivative of $V_{y}$ is given by $\dot{V}_{y}=-k_{y} V_{y}$. Again, one has exponential convergence of $y(t)$ to $y_{\mathrm{d}}(t)$ :

$$
\left|y(t)-y_{\mathrm{d}}(t)\right|=\left|y(0)-y_{\mathrm{d}}(0)\right| \mathrm{e}^{-\left(k_{y} / 2\right) t} .
$$

Finally, in the design procedure Step III, the control input $u$ is chosen to be that in the control law (4).

Lemma 3. If the control input $u$ is chosen to be that in Eq. (4), the third state variable $z$ will approach $z_{\mathrm{d}}$ in Eq. (6) exponentially fast. 
Proof. Define a third Lyapunov function candidate $V_{z}=\frac{1}{2}\left(z-z_{\mathrm{d}}\right)^{2} \geq 0$. From Eqs. (3) and (4), one can verify, although tediously, that the time derivative of $V_{z}$ is given by $\dot{V}_{z}=-k_{z} V_{z}$; hence,

$$
\left|z(t)-z_{\mathrm{d}}(t)\right|=\left|z(0)-z_{\mathrm{d}}(0)\right| \mathrm{e}^{-\left(k_{z} / 2\right) t} .
$$

The following theorem summarizes the above three Lemmas, which states that the control law (4) globally stabilizes the system to the reference state

$$
\left(x_{\mathrm{r}}, y_{\mathrm{r}}, z_{\mathrm{r}}\right)=\left(x_{\mathrm{r}}, f\left(x_{\mathrm{r}}\right), f\left(x_{\mathrm{r}}\right)-x_{\mathrm{r}}\right) .
$$

Theorem. Consider the modified Chua's circuit system (1)-(3) and the feedback control (4). Given any initial condition $x(0), y(0)$, and $z(0)$, the system state converges to the reference state in Eq. (7) exponentially fast.

Proof. The stability of the controlled system is a direct consequence of Lemmas 1-3. Since in proving Lemmas 1-3, one makes no constraints on the initial condition $x(0), y(0)$, and $z(0)$, this stability result is global.

Note that in Eq. (7) the reference state $y_{\mathrm{r}}$ is obtained by substituting $x=x_{\mathrm{r}}$ in Eq. (5), and $z_{\mathrm{r}}$ is obtained by substituting $x=x_{\mathrm{r}}$ and $y=f\left(x_{\mathrm{r}}\right)$ in Eq. (6).

\section{Numerical simulations}

In this Section, we use computer simulation to verify the performance of the proposed controller, and the results are compared with those in [10].

Consider the system (1) with the initial condition $(x(0), y(0), z(0))=(0.65,0,0)$. When the reference state is set at the origin; i.e., $\left(x_{\mathrm{r}}, y_{\mathrm{r}}, z_{\mathrm{r}}\right)=(0,0,0)$, the controller in [10] cannot stabilize the system. Fig. 1 shows the controlled state response (with a control gain $k_{\mathrm{p}}=5$ ), which remains oscillatory after the control is turned on at

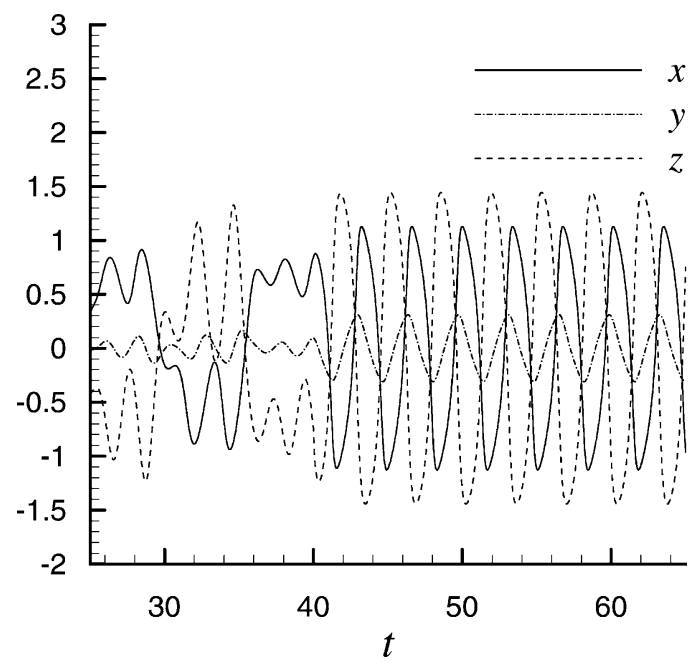

Fig. 1. State response with linear control [10]. 


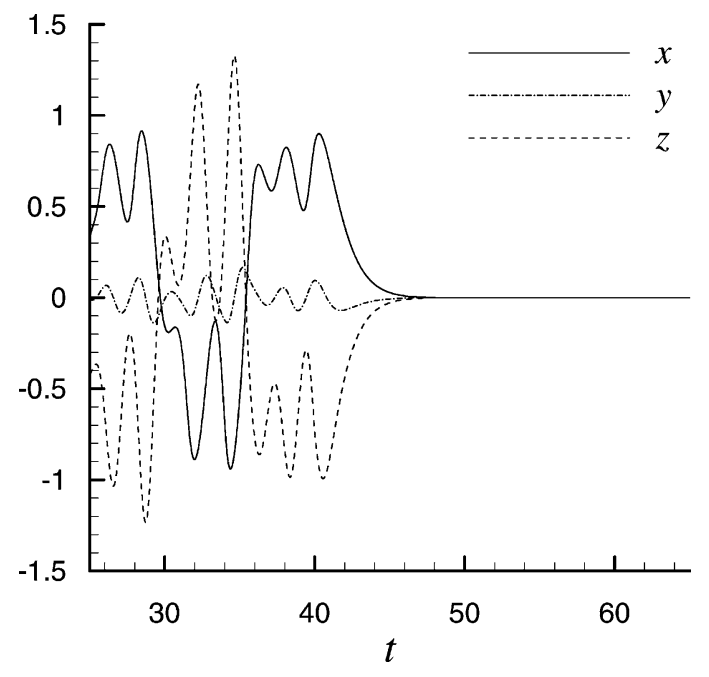

Fig. 2. State response with backstepping control.

$t=40 \mathrm{~s}$. In contrast, the backstepping control (4) proposed in this paper with $k_{x}=k_{y}=k_{z}=2$ can successfully bring the state to the origin in about $7 \mathrm{~s}$. The results are shown in Fig. 2, where similarly the backstepping control is turned on at $t=40 \mathrm{~s}$.

To further verify the effectiveness of the proposed control design, the backstepping control (4) is simulated with different control gains $k_{i}=1,1.3,2 ; i=x, y, z$. Fig. 3 shows the state norm $\|\vec{X}(t)\|=\sqrt{x^{2}(t)+y^{2}(t)+z^{2}(t)}$ versus the time $t$. It is observed that, as predicted by the analysis in Lemmas $1-3$, the state convergence rate is determined by the control gains $k_{i}$. The larger the control gains are, the faster the convergence rate is.

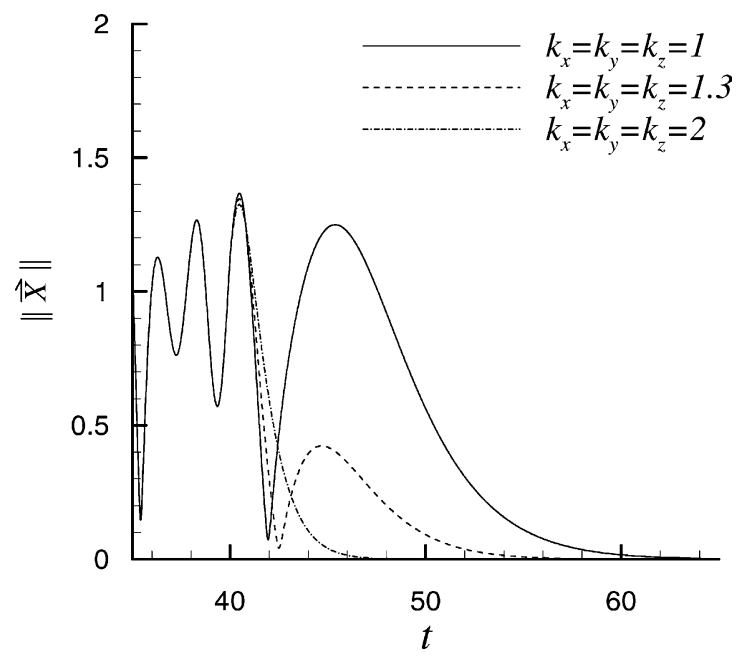

Fig. 3. Backstepping control with different gains. 


\section{Conclusions}

The control of chaos may be achieved by open-loop control (non-feedback control) or by feedback control. The advantage of using feedback control is that one can bring the system state away from chaotic motion and into any desired fixed point. Furthermore, by employing the newly developed nonlinear feedback controls, the fixed point in the reshaped state space can possess global stability, which is impossible to attain by previously suggested linear controls. This paper demonstrates the effectiveness of nonlinear control by applying the nonlinear backstepping control to suppress the chaotic motion of the modified Chua circuit system, and reshape the entire state space into one with a globally stable fixed point.

\section{References}

[1] Y. Braiman, I. Goldhirsch, Taming chaotic dynamics with weak periodic perturbations, Phys. Rev. Lett. 66 (1991) $2545-2548$.

[2] R. Lima, M. Pettini, Suppression of chaos by resonant parametric perturbations, Phys. Rev. A 41 (1990) 726-733.

[3] E. Ott, C. Grebogi, J.A. Yorke, Controlling chaos, Phys. Rev. Lett. 64 (1990) 1196-1199.

[4] U. Dressler, G. Nitsche, Controlling chaos using time delay coordinates, Phys. Rev. Lett. 68 (1992) 1-4.

[5] C.T. Chen, Linear System Theory and Design, Holt, Rinehart \& Winston, New York, 1984.

[6] J.E. Slotine, W. Li, Applied Nonlinear Control, Prentice-Hall, London, 1991.

[7] R.N. Madan (Ed.), Chua's Circuit: A Paradigm for Chaos, World Scientific, Singapore, 1993.

[8] T.T. Hartley, The duffing double scroll, in: Proceedings of the American Control Conference, Pittsburgh, PA, June 1989, pp. $419-423$.

[9] T.T. Hartley, F. Mossayebi, Control of Chua's circuit, J. Circ. Syst. Comput. 3 (1993) 173-194.

[10] C.C. Hwang, H.Y. Chow, Y.K. Wang, A new feedback control of a modified Chua's circuit system, Physica D 92 (1996) 95-100.

[11] G. Chen, X. Dong, From chaos to order-perspectives and methodologies in controlling chaotic nonlinear dynamical systems, Int. J. Bifurc. Chaos 3 (1996) 1363-1409.

[12] M. Krstić, I. Kanellakopoulos, P. Kokotović, Nonlinear and Adaptive Control Design, Wiley, New York, 1995. 